\title{
Neutrinos in Anomaly Mediated Supersymmetry Breaking with R-parity Violation
}

\section{Oscar Eboli* ${ }^{* \dagger}$}

Universidade de Sao Paulo, Brasil

E-mail: eboli@fma.if.usp.br

We show that a supersymmetric standard model exhibiting anomaly mediated supersymmetry breaking and bilinear $R$-parity violation interactions can generate the observed neutrino mass spectrum and mixings. In this model, one of the neutrinos gets its mass due to the tree level mixing with the neutralinos induced by the $R$-parity violating interactions while the other two neutrinos acquire their masses through radiative corrections.

International Europhysics Conference on High Energy Physics

July 21 st - 27th 2005

Lisboa, Portugal

* Speaker.

†Work done in collaboration with M.A. Díaz, F de Campos, R.A. Lineros, M.B. Magro and P.G. Mercadante 


\section{The AMSB-BRpV model}

There have been many experimental results in neutrino physics [1] which have established the pattern of neutrino oscillation and masses, clearly requiring physics beyond the standard model (SM) to explain it. It has been suggested a long time ago that supersymmetry and neutrino masses and mixings may be deeply tied together [2]. One way of giving mass to the neutrinos in supersymmetric models is via Bilinear $R$-Parity Violation (BRpV): in such model, bilinear terms that violate lepton number as well as $R$-Parity are introduced in the superpotential [3]. As a consequence, one neutrino acquires mass at tree level due to a low energy see-saw mechanism in which neutrinos mix with neutralinos. The other two neutrinos become massive via one-loop corrections to the neutralino-neutrino mass matrix [4].

It has been shown in [5] that BRpV can be successfully embedded into models with anomalymediated SUSY breaking scenario (AMSB) [6] giving rise at tree level to a neutrino mass compatible with the atmospheric neutrino mass scale. Here, we generalize this model including lepton number violating interaction in the three generations and we show that the inclusion of one-loop corrections to the neutralino-neutrino mass matrix leads to a neutrino spectrum and mixings compatible with the available data [7]. This is non-trivial since the radiative corrections depend on the SUSY spectrum and it is not clear a priori that the corrections will have the required properties after we impose the existing limits on the SUSY mass spectrum.

The superpotential of our BRpV model includes three $\varepsilon_{i}(i=1,2,3)$ parameters with units of mass not present in the MSSM [3]

$$
W=W_{Y}-\mu \widehat{H}_{d} \widehat{H}_{u}+\varepsilon_{i} \widehat{L}_{i} \widehat{H}_{u},
$$

where $W_{Y}$ includes the Yukawa interactions. The $\varepsilon$ terms violate lepton number and $R$-Parity and satisfy $\left|\varepsilon_{i}\right| \ll \mu$. In addition to the $\varepsilon_{i}$ terms in the superpotential, we must add the soft lagrangian bilinear terms proportional to $B_{i} \varepsilon_{i}$. For our purposes the relevant terms are

$$
V_{\text {soft }} \ni-B_{0} \mu H_{d} H_{u}+B_{i} \varepsilon_{i} L_{i} H_{u}+m_{H_{d}}^{2} H_{d}^{*} H_{d}+m_{L_{i}}^{2} \widetilde{L}_{i}^{*} \widetilde{L}_{i}
$$

where $B_{0}$ is the usual Higgs mixing term already present in the MSSM, and $B_{i}$ are the analogous bilinear terms that mix sleptons with Higgs bosons. The scalar potential of BRpV models is such that the sneutrino fields acquire a non zero vacuum expectation value $v_{i}(i=1,2,3)$ that leads to the generation of neutrino mass and mixing angles [4].

The parameters defining our BRpV-AMSB model are the usual ones in AMSB models

$$
m_{0}, m_{3 / 2}, \tan \beta, \text { and } \operatorname{sign}(\mu),
$$

where the scalar mass $m_{0}$ and the gravitino mass $m_{3 / 2}$ are given at the unification scale. This set of parameters is supplemented by the six $\mathrm{BRpV}$ parameters $\varepsilon_{i}$ and $B_{i}$. It is advantageous to trade $B_{i}$ by a parameter more directly connected to the neutrino physics observables, therefore, we choose the parameters $\Lambda_{i}$ defined as

$$
\Lambda_{i}=\mu v_{i}+\varepsilon_{i} v_{d}
$$

instead of $B_{i}$ as input parameters. One of the virtues of AMSB models is that the $S U(2) \otimes U(1)$ symmetry is broken radiatively by the running of the parameters from the GUT scale to the weak scale. This feature is preserved in our extension of AMSB models by adding BRpV. 
In the AMSB-BRpV framework, the lightest neutralino presents leptonic decays $\tilde{\chi}_{1}^{0} \rightarrow v \ell^{+} \ell^{\prime-}$, semi-leptonic ones $\tilde{\chi}_{1}^{0} \rightarrow v q \bar{q}$ or $\ell q \bar{q}$, and the invisible mode $\tilde{\chi}_{1}^{0} \rightarrow v v v$. If its decay occurs inside the detector we have to take into account new topologies in the search for SUSY since the missing transverse energy is reduced as well as there is a larger production of leptons and jets [8]. Therefore, this model is falsifiable at colliders.

\section{Reference Scenario}

Here we present a point in the parameter space which satisfies all the collider and neutrino physics constraints and then explore the parameter space around it. First, we choose an AMSB scenario in which all superpartner masses satisfy the present experimental bounds and where we obtain a correct electroweak symmetry breaking; for further details see [5]. For the AMSB parameters, we chose ${ }^{1}$

$$
m_{3 / 2}=35 \mathrm{TeV}, \quad m_{0}=250 \mathrm{GeV}, \quad \tan \beta=15, \quad \text { and } \quad \operatorname{sign}(\mu)>0 .
$$

Second, we randomly varied the parameters $\varepsilon_{i}$ and $\Lambda_{i}$ looking for solutions in which the neutrino physics restrictions given in [9] are satisfied within $1 \sigma$. An example of these solutions is

$$
\begin{aligned}
\varepsilon_{1}=0.016 \mathrm{GeV}, & \varepsilon_{2}=-0.050 \mathrm{GeV}, & \varepsilon_{3}=0.0028 \mathrm{GeV}, \\
\Lambda_{1}=-0.033 \mathrm{GeV}^{2}, & \Lambda_{2}=-0.081 \mathrm{GeV}^{2}, & \Lambda_{3}=-0.090 \mathrm{GeV}^{2} .
\end{aligned}
$$

The neutrino parameters obtained in this point of the parameter space are

$$
\begin{aligned}
\Delta m_{\mathrm{atm}}^{2}=2.0 \times 10^{-3} \mathrm{eV}^{2}, & \Delta m_{\mathrm{sol}}^{2}=8.2 \times 10^{-5} \mathrm{eV}^{2}, \\
\tan ^{2} \theta_{\mathrm{atm}}=1.23, & \tan ^{2} \theta_{\mathrm{sol}}=0.40, \quad \tan ^{2} \theta_{13}=0.041,
\end{aligned}
$$

which agree with the present experimental results.

In order to understand the dependence of the neutrino mass squared differences and mixing upon the model parameters we must study the effective neutrino mass matrix which receives tree level and one-loop contributions in our model [5]. In general, the effective neutrino mass matrix at one-loop has the approximate form $\mathbf{M}_{i j}^{\mathrm{eff}}=A \Lambda_{i} \Lambda_{j}+B\left(\varepsilon_{i} \Lambda_{j}+\varepsilon_{j} \Lambda_{i}\right)+C \varepsilon_{i} \varepsilon_{j}$, where the coefficient $A$ receives tree-level as well as one-loop contributions, and $B$ and $C$ are one-loop generated.

As a general rule, the solar mass squared difference is more sensitive to parameters changes than the atmospheric one since it is generated by radiative corrections; for further details see [5]. As an illustration, we present in Fig. 1 the $\Delta m_{\text {solar(atm) }}^{2}$ dependence upon $m_{0}$ and $\varepsilon_{2}$. As we can see from this figure, the atmospheric mass squared difference is within the experimentally allowed region in a larger range of $m_{0}\left(\varepsilon_{2}\right)$ than the solar $\Delta m^{2}$.

\section{Acknowledgments}

This work was supported in part by Conselho Nacional de Desenvolvimento Científico e Tecnológico (CNPq), and by Fundação de Amparo à Pesquisa do Estado de São Paulo (FAPESP).

\footnotetext{
${ }^{1}$ This solution is different from the one presented in [5].
} 

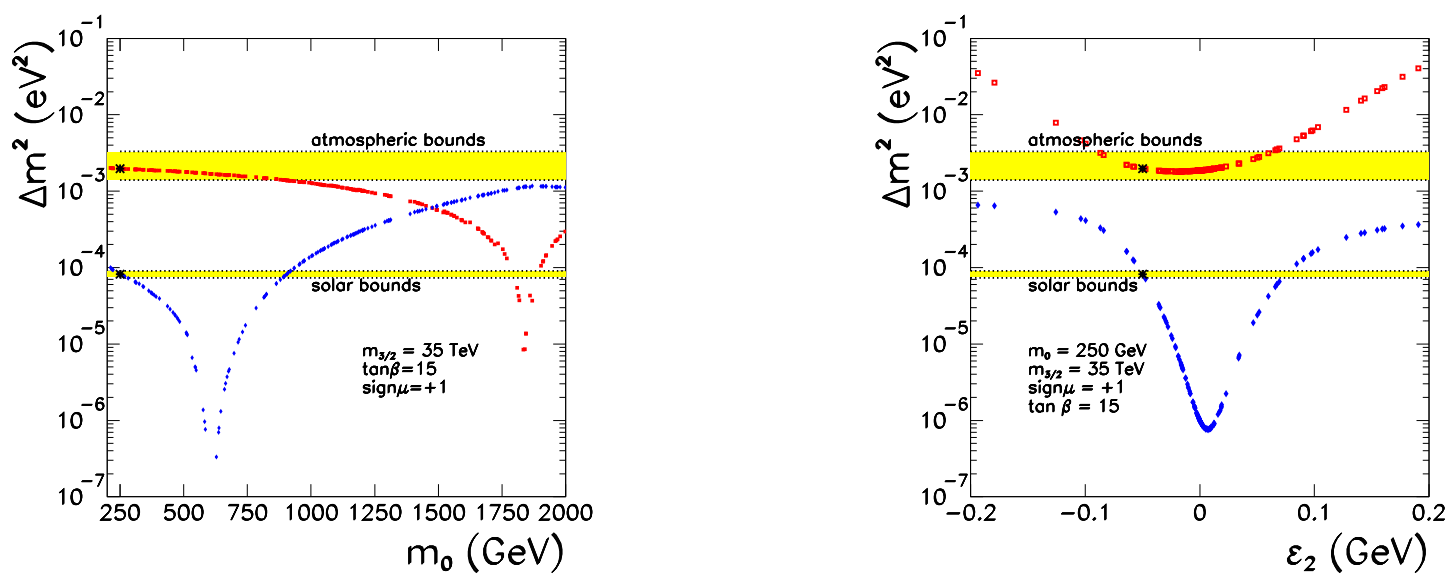

Figure 1: $\Delta m_{\text {solar(atm) }}^{2}$ dependence upon $m_{0}$ (left panel) and $\varepsilon_{2}$ (right panel). We present the $1 \sigma$ limits on these quantities as bands. The star represents our reference point.

\section{References}

[1] Q. R. Ahmad et al. [SNO Coll.], Phys. Rev. Lett. 89, 011302 (2002); S. Fukuda et al. [Super-Kamiokande Coll.], Phys. Lett. B 539, 179 (2002); K. Eguchi et al. [KamLAND Coll.], Phys. Rev. Lett. 90, 021802 (2003); M. H. Ahn et al. [K2K Coll.], Phys. Rev. Lett. 90, 041801 (2003).

[2] C. S. Aulakh and R. N. Mohapatra, Phys. Lett. B 119, 13 (1982); L. J. Hall and M. Suzuki, Nucl. Phys. B 231, 41 (1984); G. G. Ross and J. W. F. Valle, Phys. Lett. B 151, 375 (1985); J. R. Ellis, G. Gelmini, C. Jarlskog, G. G. Ross and J. W. F. Valle, Phys. Lett. B 150, 142 (1985).

[3] R. Hempfling, Nucl. Phys. B 478, 3 (1996); M. Nowakowski and A. Pilaftsis, Nucl. Phys. B 461,19 (1996); A. S. Joshipura and M. Nowakowski, Phys. Rev. D 51, 2421 (1995); T. Banks, Y. Grossman, E. Nardi and Y. Nir, Phys. Rev. D 52, 5319 (1995); H. P. Nilles and N. Polonsky, Nucl. Phys. B 484, 33 (1997).

[4] M. Hirsch, et al., Phys. Rev. D 62, 113008 (2000) [Erratum-ibid. D 65, 119901 (2002)]; J. C. Romão, et al., Phys. Rev. D 61, 071703 (2000).

[5] F. De Campos, M. A. Díaz, O. J. P. Éboli, M. B. Magro and P. G. Mercadante, Nucl. Phys. B 623, 47 (2002); M. A. Díaz, R. A. Lineros and M. A. Rivera, Phys. Rev. D 67 (2003) 115004.

[6] L. Randall and R. Sundrum, Nucl. Phys. B 557, 79 (1999); G. F. Giudice, M. A. Luty, H. Murayama and R. Rattazzi, JHEP 9812, 027 (1998).

[7] F. de Campos, O. J. P. Eboli, M. B. Magro, W. Porod, D. Restrepo and J. W. F. Valle, Phys. Rev. D 71, 075001 (2005) [arXiv:hep-ph/0501153].

[8] M. B. Magro, F. de Campos, O. J. P. Éboli, W. Porod, D. Restrepo and J. W. F. Valle, JHEP 0309, 071 (2003).

[9] See, for instance, M. Maltoni, T. Schwetz, M. A. Tortola and J. W. F. Valle, Phys. Rev. D 67, 013011 (2003); M. C. Gonzalez-García and Y. Nir, Rev. Mod. Phys. 75, 345 (2003). 